\title{
Haemodynamic instability in a critically ill patient with covid-19 pneumonia: searching over the chest - report of a clinical case and mini-review of the literature
}

\author{
Simone Conci ${ }^{*}$, Andrea Ruzzenente ${ }^{1}$, Katia Donadello ${ }^{2}$, Adam J Cybulski ${ }^{3}$, Corrado Pedrazzani ${ }^{1}$, Tommaso Campagnaro ${ }^{1}$, Vittorio \\ Schweiger $^{2}$, Andrea Dalbeni ${ }^{4}$, Giancarlo Mansueto ${ }^{3}$, Enrico Polati ${ }^{2}$ and Alfredo Guglielmi ${ }^{1}$ \\ ${ }^{1}$ Department of Surgery, Division of General and Hepatobiliary Surgery, G.B. Rossi University Hospital, University of Verona, Verona, Italy \\ ${ }^{2}$ Department of Surgery, Division of Anaesthesia and Intensive Care B, G.B. Rossi University Hospital, University of Verona, Verona, Italy \\ ${ }^{3}$ Department of Radiology, G.B. Rossi University Hospital, University of Verona, Verona, Italy \\ ${ }^{4}$ Department of Medicine, Division of General Medicine and Hypertension, COVID-Unit, G.B. Rossi University Hospital, University of Verona, Verona, Italy
}

\begin{abstract}
A 77-year-old male with multiple comorbidities developed COVID-19 pneumonia with severe respiratory distress and required hospitalization and subsequent transfer to the intensive care unit (ICU). The patient was treated according to the COVID-19 Critically Ill Protocol and required mechanical ventilation in both supine and prone positions as well as antimicrobial therapies and deep venous thrombosis prophylaxis. Fourteen days after ICU admission, the patient developed haemodynamic instability with a decrease in haemoglobin levels and renal function deterioration. The contrast-enhanced computed tomography (CT) scan revealed a large retroperitoneal haematoma with signs of active bleeding. The patient underwent surgical removal of the haematoma and haemostatic packing. Spontaneous retroperitoneal bleeding $(\mathrm{SRB})$ is a rare but potentially lethal event with unspecific clinical manifestations that may lead to both misdiagnosis and delayed treatment. $\mathrm{SRB}$ can be a potentially fatal complication of anticoagulation therapy. However, in 10-15\% of cases, it may also occur without an association with anticoagulant or antiplatelet regimens. Approximately half of patients require invasive management, such as interventional radiology (IR) procedures or surgery in cases of concurrent surgical conditions, abdominal compartment syndrome or failure of previous IR procedures.

Anticoagulant therapies are used in critically ill patients with COVID-19 pneumonia to reduce mortality due to thromboembolic events. However, the need for mechanical ventilation and prone positioning in association with anticoagulant/antithrombotic therapies may lead to an increased risk of spontaneous bleeding. SRB should always be taken into account in critically ill patients with severe COVID-19 pneumonia with haemodynamic instability without suspicion of other acute cardiovascular events.
\end{abstract}

\section{Core tip}

The real incidence of bleeding in COVID-19 patients is unknown due to the poor knowledge on the pathophysiology of this new virus and to the lack of data reported in the literature. In particular, patients admitted to the intensive care unit (ICU) with severe pneumonia who require mechanical ventilation in the prone position and the administration of anticoagulant/antithrombotic therapies may be subjected to an increased risk of spontaneous bleeding. We report a mini-review and a case of spontaneous retroperitoneal bleeding (SRB) in a critically ill patient with COVID-19 pneumonia. SRB should be suspected in critically ill patients with haemodynamic instability without suspicion of other acute cardiovascular events.

\section{Clinical Case}

A 77-year-old male came to our hospital with persistent fever and cough 3 days after returning from travel to the Red Sea, Egypt. He had a medical history of well-controlled hypertension, dyslipidaemia and chronic atrial fibrillation treated with atorvastatin and flecainide/ apixaban, respectively. On clinical examination, he was breathless and tachypnoeic ( 28 breaths per $\mathrm{min}$ ), his temperature was $38.5^{\circ} \mathrm{C}$, and oxygen saturation was $88 \%$ in room air. Laboratory investigation showed a C-reactive protein level of $147 \mathrm{mg} / \mathrm{L}$ (normal <5), a haemoglobin concentration of $14.3 \mathrm{mg} / \mathrm{dL}$ (normal 13.5-16.0), a white blood cell count of $8.25 \times 109$ per L (normal 4.3-10.0), an activated partial thromboplastin time (aPTT) of 1.2 (normal 0.80-1.20), and an international normalized ratio (INR) of 1.52 (normal 0.82-1.17); arterial blood gas analysis revealed respiratory alkalosis and hypoxemia with a $\mathrm{pH}$ of 7.51 (normal 7.35-7.45), a CO2 pressure of $34 \mathrm{mmHg}$ (normal 35-40), an oxygen pressure of $42 \mathrm{mmHg}$ (normal 80-100), and a lactate concentration of $1.2 \mathrm{mmol} / \mathrm{L}$ (normal 0.5-2.0). SARS-CoV-2 was detected in both mid-turbinate and throat swabs by PCR, and COVID-19 was confirmed by sequencing.

${ }^{\star}$ Correspondence to: Simone Conci, Department of Surgery, Division of General and Hepatobiliary Surgery, University of Verona Medical School, G.B. Rossi University Hospital, Piazzale L.A. Scuro, 10, Verona 37134, Italy, Tel: +39045-8124655; Fax: +39-045-8027426; E-mail: simone.conci@aovr.veneto.it

Key words: spontaneous Retroperitoneal Bleeding; Spontaneous Retroperitoneal Haematoma; COVID-19; SARS-CoV-2; Anticoagulant Therapies; Haemostatic packing

Received: September 09, 2020; Accepted: September 21, 2020; Published: September 24, 2020 
One day after hospital admission, the patient developed severe respiratory distress that required his transfer to the intensive care unit (ICU).

During his ICU stay, he was treated according to the COVID-19 Critically Ill Patient Protocol: after rapid sequence intubation (RSI), he received continuous sedation and neuro-muscular paralysis infusion, and he was ventilated with the mandatory volumetric mode with a small tidal volume ( $6 \mathrm{ml} / \mathrm{kg}$ of ideal body weight); best PEEP was repeatedly measured with the open-lung approach both in the supine and the prone positions. The prone position was maintained for 14-20 hours/day, with sedation/paralysis windows performed daily for proper neurological evaluation. Enteral nutritional support was targeted at $25 \mathrm{Kcal} / \mathrm{kg} /$ day with supplemental vitamins and oligo-elements, and glycaemia was controlled with continuous insulin infusion; anti-microbic treatment involved 10-day oral hydroxychloroquine and lopinavir/ ritonavir, compassionate use of remdesivir for 10 days, initial empiric ceftriaxone and levofloxacin; urine pneumococcal antigen turned positive, and Gram positive cocci were found in the admission blood cultures. Vancomycin was thus added to the treatment while waiting for microorganism identification. Deep venous thrombosis prophylaxis was guaranteed with $100 \mathrm{UI} / \mathrm{kg}$ of enoxaparin once/day. After a week of being in a critical condition, the patient started to recover, and he was extubated 11 days after ICU admission. Nevertheless, 3 days later, 14 days after ICU admission, the patient developed sudden haemodynamic instability with a sharp decrease in haemoglobin concentration (from 9.5 to $6.6 \mathrm{~g} / \mathrm{dL}$ ) and renal function deterioration.

At clinical examination, there was palpable left abdominal quadrant tenderness. Other laboratory investigations showed a aPTT of 1.25 (normal 0.80-1.20), an INR of 1.60 (normal 0.82-1.17), a creatinine level of $144 \mu \mathrm{mol} / \mathrm{L}$ (normal 53-115), and an estimated glomerular filtration rate (eGFR) of $40 \mathrm{ml} / \mathrm{min} / 1.73 \mathrm{~m} 2$ (normal > 60). A contrastenhanced computed tomography (CT) scan of his abdomen revealed a retroperitoneal haematoma involving the left psoas muscle pushing the left colon and the left kidney forward and medially (Figure 1A-1C) with signs of active contrast extravasation (Figure $1 \mathrm{~A}$ and $\mathrm{C}$ arrows). Considering the multiple bleeding sources, critical haemodynamic instability and signs of abdominal compartment syndrome (acute

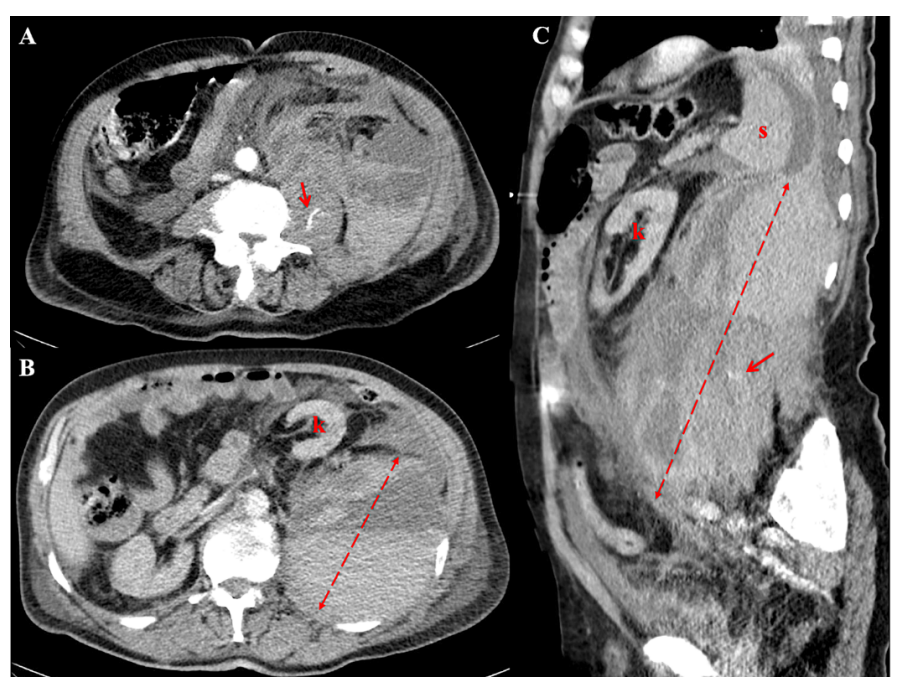

Figure 1. The contrast-enhanced computed tomography scan of the abdomen revealing a large retroperitoneal haematoma involving the left psoas muscle pushing the left colon and the left kidney forward and medially (A-C) with signs of contrast extravasation (arrows, A and C)

S, spleen; K, kidney renal failure, intra-abdominal pressure of $22 \mathrm{mmHg}$ ), the patient was a candidate for urgent surgical exploration. The haematoma was evacuated, and diffuse active bleeding from the left psoas muscle, lateral abdominal wall and retroperitoneal tissue was found. Haemostasis stitches were placed on the main active bleeding sources, but due to persistent wide blood dripping and unsatisfactory bleeding control, haemostatic packing needed to be carried out. The patient requested haemodynamic support with vasopressors for the following 72 hours, various transfusions with red blood cells and fresh frozen plasma, and continuous haemodiafiltration. After a week and two surgical revisions with lap pad changing, the packing was definitely removed. The patient was extubated 10 days after spontaneous retroperitoneal bleeding and discharged from the ICU 6 days thereafter.

\section{Spontaneous Retroperitoneal Bleeding and critical issues in COVID-19 patients}

Spontaneous retroperitoneal bleeding (SRB) is a rare but potentially lethal event with unspecific clinical manifestations that may lead to both misdiagnosis and delayed treatment. Its etiopathogenesis may rely on either parenchymal (adrenal or renal masses) or vascular origin (rupture of splanchnic vessels). Vascular SRB is seen in patients with arterial aneurysms or malformations, in patients undergoing anticoagulation therapy, in those with coagulative cascade deficits, or in those on renal replacement treatment $[1,2]$. SRB represents a potentially fatal complication of anticoagulation therapy, with reported incidence and mortality rates of $0.6-6.6 \%$ and $10-20 \%$, respectively $[3,4]$. It commonly involves the psoas or gluteal muscles, and all types of anticoagulation therapy have been implicated, both anticoagulant and antiplatelet drugs. Contrary to common belief, supratherapeutic anticoagulation is implied only in one-third of the cases of SRB. Moreover, $10-15 \%$ of the described patients were not on anticoagulant or antiplatelet regimens [5]. These findings highlight the possible participation of occult vasculopathy, unrecognized minor trauma, or muscular strain in the pathogenesis of SRB.

CT is the imaging modality of choice when SRB is suspected. It is useful to delineate the anatomical extension and size of the haematoma in order to evaluate the effect of SRB-related mass effects; furthermore, it may identify the bleeding origin and show signs of active bleeding through the presence of contrast extravasation [6].

The present evidence for the best SRB management is based only on small cohort series or isolated case reports; however, the treatment strategy may be summarized as "support the volume and stop the bleeding". Approximately half of the patients described in the available literature were successfully managed with a medical and non-invasive treatment that included intravenous fluid administration with crystalloids and colloids, vasopressor support and blood product transfusion in case of massive bleeding with severe hypotension [1,4,7-9]. Specific drugs to reverse coagulopathy can be considered when indicated (i.e., vitamin $\mathrm{K}$, protamine sulfate, prothrombin complex concentrates, recombinant factor VIII and IX) [7,8]. Invasive treatment (interventional radiology procedures and surgery) should be considered in cases of persistent haemodynamic instability with active bleeding sources on CT imaging or in cases of significant abdominal or retroperitoneal compressive symptoms (abdominal compartment syndrome). Interventional radiology (IR) procedures with selective arterial embolization were indicated in cases of evident arterial extravasation on CT scans and were successfully performed in approximately half of the cases. Surgery was reserved for patients with failed IR procedures, concurrent surgical conditions, or significant 

the literature

abdominal hypertension symptoms due to haematoma enlargement. The primary goals for surgery are first to control all active bleeding sources and then to remove the large haematoma. Nevertheless, the removal of the haematoma may increase bleeding by removing its tamponade effect. When persistent wide bleeding or unsatisfactory control was obtained, packing with lap pads may be the only surgical option. In these cases, the retroperitoneum may need to be packed and re-explored at 24-48 $\mathrm{h}[1,4,5,9]$.

Early detection and management of SRB may be of particular interest now that the emerging spread of COVID-19 has significantly increased the number of critically ill patients worldwide.

Viral infections, such as SARS-CoV-2, seem to induce a hypercoagulable state due to endothelial dysfunction that results in both increased thrombin generation and reduced fibrinolysis $[10,11]$.

As clinical validation of these theories, a recent study on lung dissection described the presence of occlusion and micro-thrombosis formation in pulmonary small vessels of a critically ill patient with COVID-19 pneumonia [12]. Moreover, Cui S et al reported a $25 \%$ incidence of venous thromboembolism in ICU patients with severe COVID-19 pneumonia without thromboprophylaxis, with a mortality rate of $40 \%$ [13].

Therefore, early administration of anticoagulant therapy in patients with severe COVID-19 pneumonia has been suggested for improving outcomes, especially in those who meet the criteria for sepsis-induced coagulopathy (SIC score) [14]; however, a protocol with specific inclusion or exclusion criteria and a standardized anticoagulation regimen is still under debate.

According to the published recommendations from the International Society of Thrombosis and Hemostasis (https://www. isth.org), from the American Society of Hematology (https://www. hematology.org/covid-19) and from the Society for Thrombosis and Haemostasis Research (http://gth-online.org), hospitalized patients with COVID-19 pneumonia should receive pharmacological thromboprophylaxis according to a risk stratification score, with lowmolecular-weight heparin (LMWH) or unfractionated heparin (UFH) according to renal function, unless contraindicated.

Furthermore, the spread of SARS-CoV-2 infection in Western countries has led to the contagion and hospitalization of thousands of patients, particularly elderly patients with multiple comorbidities, including those requiring the use of oral anticoagulants, such as patients with prosthetic heart valves and with non-valvular chronic atrial fibrillation, and for the prevention and treatment of venous thromboembolism [15]. The development of COVID-19 pneumonia in anticoagulated patients and their need for ICU admission expose them to peculiar issues not only related to acute viral infection but also to their specific management.

Some authors suggested the direct switch of oral anticoagulant therapies to subcutaneous heparin at therapeutic dosages, while others considered parenteral heparin a better choice for personalized control and treatment [16].
Currently, there is a lack of data reporting the real incidence of bleeding in COVID-19 patients, particularly in ICU patients with severe pneumonia; however, the need for mechanical ventilation and prone position in association with anticoagulant/antithrombotic therapies may lead to an increased risk of spontaneous bleeding. SRB should always be taken into account in critically ill patients with severe COVID-19 pneumonia with haemodynamic instability without suspicion of other acute cardiovascular events.

\section{References}

1. Sahu KK, Mishra AK, Lal A, George SV, Siddiqui AD (2020) Clinical spectrum, risk factors, management and outcome of patients with retroperitonealhematoma: a retrospective analysis of 3-year experience. Expert Rev Hematol 3: 1-11. [Crossref]

2. Gutowski ED, Tio MC, Burch EA, Leaf DE (2020) Peritoneal dialysate tamponading a massive retroperitoneal hemorrhage. Kidney Int 97: 810. [Crossref]

3. Salemis NS, Oikonomakis I, Lagoudianakis E, Boubousis G, Tsakalakis C, et al. (2014) Enoxaparin induced spontaneous massive retroperitoneal hematoma with fatal outcome. Am J Emerg Med 32: 1559. [Crossref]

4. Gonzalez C, Penado S, Llata L, Valero C, Riancho JA (2003) The clinical spectrum of retroperitoneal hematoma in anticoagulated patients. Medicine 82: 257-262. [Crossref]

5. Sunga KL, Bellolio MF, Gilmore RM, Cabrera D (2012) Spontaneous retroperitoneal hematoma: etiology, characteristics, management, and outcome. J Emerg Med 43: e157-e161. [Crossref]

6. Badea R, Chiorean L, Mitre C, Botar-Jid C, Caraiani C (2013) Spontaneous retroperitoneal and subcapsular liver hematoma. The diagnostic contribution of CT, US and CEUS. Case report. Med Ultrason 15: 157-160. [Crossref]

7. Wilsey HA, Bailey AM, Schadler A, Davis GA, Nestor M, et al. (2020) Comparison of Low- Versus High-Dose Four-Factor Prothrombin Complex Concentrate (4F-PCC) for Factor Xa Inhibitor-Associated Bleeding: A Retrospective Study. J Intensive Care Med 3: 885066620916706. [Crossref]

8. Mishra AK, Sahu KK, Siddiqui AD, George SV (2020) Initiation of a fixed- dose fourfactor prothrombin complex concentrate protocol. J Thromb Thrombolysis 49: 332-333. [Crossref]

9. Chan YC, Morales JP, Reidy JF, Taylor PR (2008) Management of spontaneous and iatrogenic retroperitoneal haemorrhage: conservative management, endovascular intervention or open surgery? Int J Clin Pract 62: 1604-1613. [Crossref]

10. Levi M, van der Poll T (2017) Coagulation and sepsis. Thromb Res 149: 38-44 [Crossref]

11. Schmitt FCF, Manolov V, Morgenstern J, Fleming T, Heitmeier S, et al. (2019) Acute fibrinolysis shutdown occurs early in septic shock and is associated with increased morbidity and mortality: results of an observational pilot study. Ann Intensive Care 9: 19. [Crossref]

12. Menter T, Haslbauer JD, Nienhold R, Savic S, Hopfer H, et al. (2020) Post-mortem examination of COVID19 patients reveals diffuse alveolar damage with severe capillary congestion and variegated findings of lungs and other organs suggesting vascular dysfunction. Histopathology. 14134. [Crossref]

13. Cui S, Chen S, Li X, Liu S, Wang F (2020) Prevalence of venous thromboembolism in patients with severe novel coronavirus pneumonia. J Thromb Haemost 18: 1421-1424. [Crossref]

14. Tang N, Bai H, Chen X, Gong J, Li D, et al. (2020) Anticoagulant treatment is associated with decreased mortality in severe coronavirus disease 2019 patients with coagulopathy. J Thromb Haemost 18: 1094-1099. [Crossref]

15. World Health Organization (WHO) (2020) Coronavirus disease 2019 (COVID-19). Situation Report -89

16. Testa S, Paoletti O, Giorgi-Pierfranceschi M, Pan A (2020) Switch from oral anticoagulants to parenteral heparin in SARS-CoV-2 hospitalized patients. Intern Emerg Med 15: 1-3. [Crossref]

Copyright: (C2020 Conci S. This is an open-access article distributed under the terms of the Creative Commons Attribution License, which permits unrestricted use, distribution, and reproduction in any medium, provided the original author and source are credited. 